\title{
Relationship Identification \& Prophecy of Diseases Connection using Micro-Rna of Genomic Facts
}

\author{
R.Kavitha, G.Kavitha, S.Sangeetha. G.Michael
}

\begin{abstract}
The current process of finding the relationship between the father and the son and also predicting the diseases that is yet to occur is quite inaccurate because it includes only the gene-id of the respected person. In order to handle or to make this system more accurate, we propose this system by using the chromosome structure of the person. This system takes the input of the chromosome structure of the son that has been partitioned from the father's chromosome structure. It initially preprocesses the image of the son using the collaborative filtering for making it look different from the input image to show the similarity between the father and the son. It then detects the edge of the structure after preprocessing it using the SOBEL edge recognition calculation. The SOBEL edge discovery calculation is that the inclination of the picture is determined for every pixel position in the picture.After detecting the edges of those input images, matching process starts between the input image and the list of father chromosome images. Then the matched output appears. In order to predict the diseases which is yet to come in future for the son is represented graphically by dividing it into three colors, firstly green represents there is less possibility of the son getting the disease, secondly yellow represents there may be any chance of son getting the disease and finally red represents there is high possibility of son getting the disease.
\end{abstract}

KEYWORDS: Image processing, Edge detection, Image matching, Graphical representation

\section{I .INTRODUCTION}

Data mining is the way toward finding plans in expansive edifying collections including techniques at the convergence motivation behind AI, encounters, and database frameworks. It is a basic method where sharp frameworks are related with empty information plans. It is an interdisciplinary subfield of programming building. The general focus of the information mining procedure is to expel data from an edifying document and change it into a reasonable structure for further use. Other than the undesirable examination step, it wires database and data the experts' perspectives, data pre-dealing with, model and enlistment considerations, enchanting

Revised Manuscript Received on July 22, 2019

R. Kavitha, Department of CSE, Bharath Institute of Higher Education and Research, Chennai, Tamilnadu, India.

G. Kavitha, Assistant Professor, Department of Computer Science and Engineering, Bharath Institute of Higher Education and Research, Chennai, India.

S. Sangeetha, Department ofCSE, Bharath Institute of Higher Education and Research, Chennai, Tamilnadu, India.

G.Michael, Department of computer science and engineering ,Bharath Institute of Higher Education and Research,Chennai,Thamilnadu ,India. quality estimations, multifaceted nature thoughts, post-masterminding of discovered structures, affirmation, and web engaging Information mining is the examination experience of the "learning divulgence in databases" framework or KDD.

Picture handling is arranging of pictures utilizing numerical activities by a banner preparing for which the information is a picture, a development of pictures or a video, for instance, a photograph or a video structure; the yield of picture pre-planning might be either a picture or a lot of qualities or parameters identified with the picture. Most picture arranging systems fuse considering the to be as a two-dimensional pennant and applying standard flag dealing with procedures to it. Pictures are likewise dealt with as three-dimensional signs with the third estimation being time or the $\mathrm{z}$-focus point.

In recent years, the technology is being increased to greater level in several fields. This system focuses on the medical field. our system helps the doctors or the admins to predict the diseases easily with help of some tools and technologies.

In our system, we are going to find the relation of father and son using two of the factors. One is the geneid and the other is the chromosome structure. Nextly the diseases that the father has, can also occur for the son are being predicted. When dealing with geneid it was bought from the real time hospitals. Using the father's geneid, the son's geneid is bought by the way of molecular weight and the possibilities of the diseases for the son are displayed by using some of the algorithms. When dealing with chromosome structure, son's structure is given as input and the fathers structure is retrieved using the $\mathrm{cM}($ Centimorgan) and SNP (Singlenucleotide Polymorphisms) values and the diseases are predicted and the disease severity is displayed in a graph. This project not only deals with human chromosome but little process on rat chromosome structure are also being included The performance is more accurate when dealing with chromosome structure than with gene id. It also becomes more realistic when dealing the chromosome. The understandability is also very easy with chromosomes. When dealing with geneid it speaks of molecular weight which is the back-end calculation. When with chromosome structure it gives the visual similarity which the user or the admin

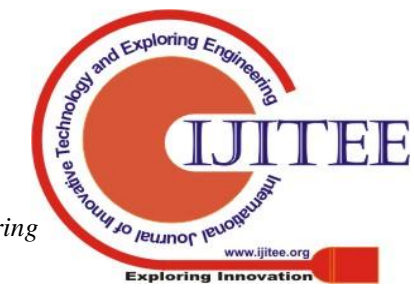


can easily view the relation between the father and the son. The geneid format differs from organization to organization but the structure is standard format for any type of chromosome.

\section{CM(CENTIMORGAN)}

Contracted $\mathrm{cm}$ is an extent of inherited linkage. Think of it as an extent of DNA information inside a chromosome. Each chromosome contains differing proportions of information. Chromosome 1 contains $281.5 \mathrm{cM}$ of information. Chromosome 2 has $263.7 \mathrm{cM}$. Chromosome 21 has recently $70.2 \mathrm{cM}$.

\section{III.SNP(SINGLENUCLEOTIDEPOLYMORPHISM)}

SNPs, or single-nucleotide polymorphisms, are unobtrusive bits of a chromosome that contain specific squares of information. There are a large number of them for every chromosome. SNPs are stood out between two people from check whether they facilitate. The proportion of information in planning SNPs is evaluated in cM.The cM regards for SNP matches are to a great extent implied as "chromosome length" or "match length". In any case, information is even more thickly squeezed in explicit districts or SNPs inside chromosomes, so there's not a prompt association between's number of SNPs and cM aggregate. When you see GEDmatch's graphical depiction of chromosome arranges, a more noteworthy organizing square does not continually mean a higher cM regard.Start and End Location.Singular markers (called base sets - the things that SNPs are made of) inside a chromosome are numbered. There are a huge number of these markers per chromosome. A section of a chromosome can be recognized by these area numbers.

\section{MICRO-RNA OF GENOMIC DATA}

Quality articulation in cells and tissues of each staggering animal is unquestionably controlled and, all things considered, subject to different conditions, (for instance, improvement, changes in nature, ailments or meds). Distinctive cells and organ structures inside such living thing (checking individuals) contain differing quality verbalization profiles, along these lines genuine understanding of managerial frameworks connected with such enunciation addresses one of the key issues in genomic medicine.

Separating miRNAs from different classes of little RNAs that are available in the phone is frequently unwieldy especially the qualification from endogenous little meddling RNAs (siRNAs). The most critical refinement among miRNAs[1] and siRNAs is whether they quietness their very own appearance. Practically all siRNAs (paying little notice to their viral or other beginning stage) calm a comparable locus from which they were resolved. On the other hand, most miRNAs don't tranquil their own one of a kind loci, yet extraordinary characteristics. miRNAs control grouped pieces of progress and physiology, consequently understanding its natural employment is showing progressively basic.[12] Examination of miRNA enunciation may give gainful information, as deregulation of its ability can incite human ailments, for instance, dangerous development, cardiovascular and metabolic diseases, liver conditions and resistant brokenness.

\section{V . LITERATURE SURVEY}

Hailin chen and zuping zhang(2018):In this paper, we proposed a miRNA-based computational strategy HNBI to induce novel medication ailment relationship for medication repositioning. Likeness estimations and tentatively bolstered affiliation data were first coordinated to develop a three-layer medicate miRNA-malady heterogeneous system. Our technique at that point refreshed the quality of load between unlinked tranquilize miRNA, miRNA-sickness, and medication illness matches iteratively till settled. In light of data on the heterogeneous system, the last load of medication malady affiliations was gotten by outlining the estimations of ways associating the two kinds of hubs. We organized the potential medication sickness relationship as indicated by the new weight. At the point when connected to the gathered informational index for cross-approval tests, our strategy demonstrated predominant execution in medication ailment affiliation forecasts contrasted and two best in class strategies. Besides, our technique HNBI joined data of target miRNAs to comprehend the components of activity of medications and the atomic instruments of maladies. A contextual investigation on the medication Terazosin showed that some anticipated signs with high positions were upheld by the ongoing writing, which further delineated the down to earth handiness of our technique. At last, far reaching expectations of relationship among medications and ailments were discharged for future medication repositioning thinks about.

Rohith Babu, I.Rakesh, Auxilia Osvin Nancy,(2018):In this paper, we propose a novel strategy called SNCoNMF (Sparse Network regularized non-negative grid factorization for Co-administrative modules recognizable proof) which embraces various nonnegative lattice factorization structure to distinguish co-administrative modules including miRNAs, TFs and qualities. This technique mutually coordinates miRNA, TF and quality articulation profiles, and extra priori systems were included a regularized way. Also, to keep away from the sparsity of these systems, we utilize the sparsity punishments to the factors to accomplish secluded arrangements. The numerical definition can be adequately comprehended by an iterative multiplicative refreshing calculation. We apply this technique to various genomic information including the articulation profiles of miRNAs, TFs and qualities on bosom malignant growth got from TCGA, priori miRNA-quality controls, TF-quality directions and quality connections. The outcomes demonstrate that the miRNAs, TFs and 
qualities of the co-administrative modules are essentially related and modules have a sensible size dissemination. Besides, the co-administrative modules are fundamentally advanced in GO organic procedures and KEGG pathways, individually.

Z. Sun, Z. Wu, F. Zhang, Q. Guo, H. Chen, J. Zhao, D. Song, Q. Huang, L. Li, and J. Xiao,(2016):Bosom disease is the most well-known reason for malignant growth demise in ladies and positions second among malignancy passings. Metastasis is the fundamental driver of death in bosom malignancy patients. In any case, the instruments hidden the intrusion and metastasis of bosom malignant growth cells remain to a great extent slippery. Here we report that the protein 9 PRAME, a tumor-related antigen separated from a melanoma, assumes a job in keeping the expansion and metastasis of bosom disease cells. Thumping down of PRAME advances bosom malignant growth cell multiplication and hinders apoptosis. What's more, hindrance of PRAME advances the attack of bosom malignancy cells. To additionally inspect the job of PRAME in vivo, we used mouse model and found the volume and the heaviness of tumors was uniquely expanded after PRAME was thumped down. This investigation exhibits that PRAME capacities as a tumor silencer in bosom disease.

M. E. Ritchie, B. Phipson, D. Wu, Y. Hu, C. W. Law, W. Shi, and G. K. Smyth,(2015): Limma is a R/Bioconductor programming bundle that gives a coordinated answer for examining information from quality articulation tests. It contains rich highlights for dealing with complex trial structures and for data acquiring to beat the issue of little example sizes. Over the previous decade, limma has been a well known decision for quality revelation through differential articulation investigations of microarray and high-throughput PCR information. The bundle contains especially solid offices for perusing, normalizing and investigating such 14 information. As of late, the capacities of limma have been altogether extended in two critical ways. In the first place, the bundle would now be able to perform both differential articulation and differential joining investigations of RNA sequencing (RNA-seq) information. All the downstream investigation apparatuses recently limited to microarray information are presently accessible for RNA-seq too. These capacities enable clients to break down both RNA-seq and microarray information with fundamentally the same as pipelines. Second, the bundle is presently ready to go past the customary quality savvy articulation investigations in an assortment of ways, breaking down articulation profiles as far as co-managed sets of qualities or as far as higher-request articulation marks. This gives upgraded potential outcomes to natural elucidation of quality articulation contrasts. This article audits the logic and plan of the limma bundle, abridging both new and recorded highlights, with an accentuation on late improvements and highlights that have not been recently depicted.
Samta Gupta, Susmita Ghosh Mazumdar(2013): Edge recognition is in the front line of picture handling for article area, it is critical to have a not too bad perception of edge recognizable proof counts. Sobel which is an outstanding edge revelation figuring is considered in this work. There exists a limit, edge's which is in the image toolbox. In the edge work, the Sobel system uses the subordinate speculation to find edges. Consequently, it returns edges at those centers where the tendency of the considered picture is generally extraordinary. The Sobel director plays out a 2-D spatial incline estimation on pictures. It uses a few dimension and vertical tendency cross sections whose estimations are $3 \times 3$ for edge ID exercises. It will moreover tell the best way to collect a Sobel pointer limit of $5 \times 5$ estimation in tangle lab to find edges.

\section{VI.OVERALARCHITECTURE}

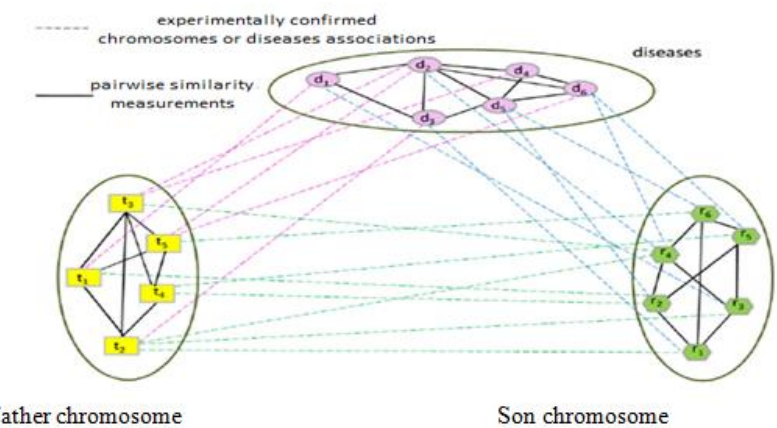

VII. METHODOLOGY

\section{A.Dataset}

The picture chromosomes of dataset have been made for examination of ailment. This dataset contains twenty two cases and five characteristics are utilized in this similar examination. The chromosome legacy and how to examining chromosomes by utilizing GEDmatch databases to discover One-to-numerous Matches, One-to-one Compare, and People who coordinate either of two kits, the ideas are relevant to comparative databases given by the DNA real testing administrations. This implies your DNA information is in the GEDmatch database so it very well may be utilized to contrast with others. Here the backend of the coding utilizing by java stage. Java is an extensively important PC programming language that is synchronous, class-based, object-masterminded, and unequivocally wanted to have as few execution conditions as could reasonably be typical. It is intended to give application originators "an opportunity to compose once, run wherever ", suggesting that collected Java code can continue running on all phases that assistance Java without the necessity for recompilation. 

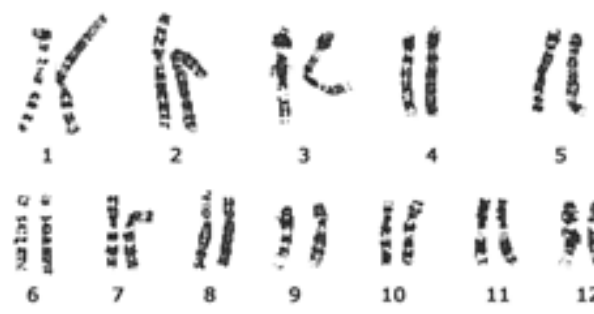

3

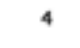

5
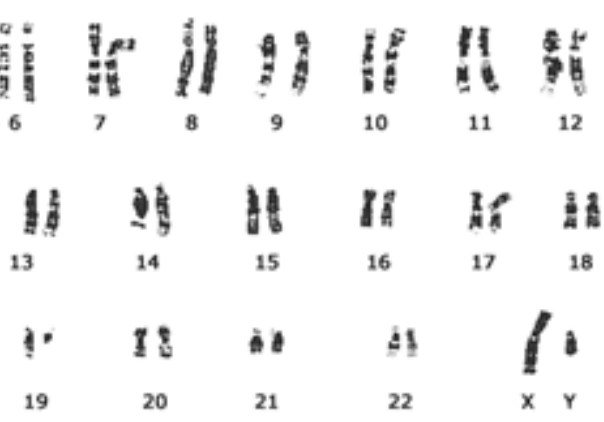

Chromosome Basics

Chromosomes are little structures found inside your cells. [13] They contain the DNA information and rules that describe your character - what you take after, how your body works, and even what genetic diseases you may have. Individuals have 46 chromosomes. In any case, chromosomes come in sets, so we customarily think of them as 23 sets of chromosomes. The underlying 22 chromosome sets (called autosomes) are numbered 1 through 22. We'll fundamentally focus on these autosomal chromosomes.

\section{B.Chromosome Inheritance}

One autosomal chromosome from each pair begins from your mother and substitute starts from your father. This infers you get half of your DNA from your mother and half from your father. Each chromosome they pass on to you is their own one of a kind mix pair of chromosomes which they got from their people (your grandparents).

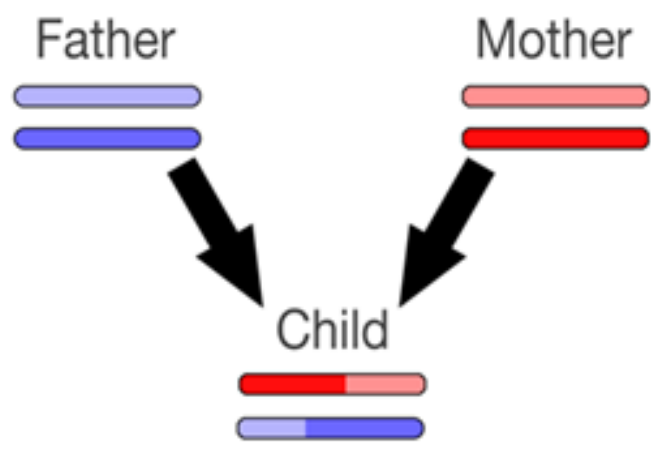

The picture above delineates how one sets of chromosomes might be passed from your folks to you. The hues don't mean anything unique - they basically portray the individual chromosomes and chromosome areas

\section{IMAGE PREPROCESSING}

Image processing is a method to perform in the use of computer algorithm to create, processing and convert signals from an image sensor in to digital image. Image processing toolbox provide a comprehensive to the set of reference in a standard algorithm work flow in a image processing to visualize perform in a image segmentation

\section{EDGE DETECTION}

Edge discovery is a picture preparing procedure for finding the limit of item inside the picture. It works by discontinuities in splendor.[8] Edge location is utilized for picture discovery information extraction in region, for example, picture preparing, $\mathrm{PC}$ form and machine rendition.

\section{IMAGE MATCHING}

Image matching is used to match the similar images from the multiple set of images with respect to the input image .It is done by a comparing the input image with the set of images and the finally produce a similar image as output. Here we have used modified greedy algorithm to match the same chromosome structure image based on some similarity between two images.

\section{GRAPHICAL REPRESENTATION}

Here we have shown the list of predicted diseases and their possibility of occurring with respect to son and is represented graphically by dividing it into three colors, firstly green represents there is less possibility of the son getting the disease, secondly yellow represents there may be any chance of son getting the disease and finally red represents there is high possibility of son getting the disease.

\section{Collaborative Filtering Algorithm}

For every client, recommender frameworks prescribe things dependent on how comparable clients loved the thing. Suppose Alice and Bob have comparable interests in computer games. Alice as of late played and delighted in the amusement Legend of Zelda: Breathe of the Wild. Sway has not played this amusement, but since the framework has discovered that Alice and Bob have comparative tastes, it prescribes this diversion to Bob. Notwithstanding client likeness, recommender frameworks can likewise perform synergistic sifting utilizing thing comparability ("Users who preferred this thing additionally loved $\mathrm{X}^{\prime \prime}$ ).

\section{Sobel Edge Detection Algorithm}

Edge acknowledgment is in the bleeding edge of picture dealing with for thing distinguishing proof, it is critical to have a better than average understanding of edge area estimations.[8] Sobel which is a predominant edge area computation is considered in this work. There exists a limit, edge.m which is in the image toolbox. In the edge work, the Sobel methodology uses the backup estimate to find edges. As such, it returns edges at those centers where the tendency of the considered picture is generally extraordinary. The Sobel chairman plays out a 2-D spatial tendency estimation on pictures. It uses several dimension and vertical point networks whose estimations are $3 \times 3$ for edge ID exercises. It will in like manner display how to amass a Sobel discoverer limit of $5 \times 5$ estimation in matlab to find edges. 


\section{F. Modified greedy Algorithm}

An adjusted excited computation called Multi-Tree-based Orthogonal Matching Pursuit (MTOMP). It is an algorithmic perspective that seeks after the basic reasoning heuristic of settling on the locally perfect choice at each stage with the objective of finding an overall perfect. In various issues, an anxious strategy does not generally create a perfect course of action, yet regardless a covetous heuristic may yield locally perfect game plans that deduced an all around perfect game plan in a reasonable proportion of time.

\section{XII .EXPERIMENTAL RESULT}

In this system a collaborative filtering is used for preprocessing method. In the preprocessing the required information that are related to the chromosome and diseases are processed. We use edge detection method to detect the edges of the chromosome structure and so that it gets the parent chromosome. For the edge detection we use the algorithm called Sobel Edge detection. This algorithm is being used because it gives the exact outer structure when compared to that of other algorithms. Then for matching purpose the Modifies Greedy algorithm is being used to match the related chromosome. Then the bar graph is being generated. From the bar graph the possibility or the chances of the diseases for the son are displayed with the percentage of diseases. The chances are also being displayed with the color differences. Then finally the histogram can be displayed for each of the input structure that is being given. Thus, by using these predictions and the bar graph, the admins and the doctors can be benefited for explaining or show casing the patients or the users to understanding an easy way.

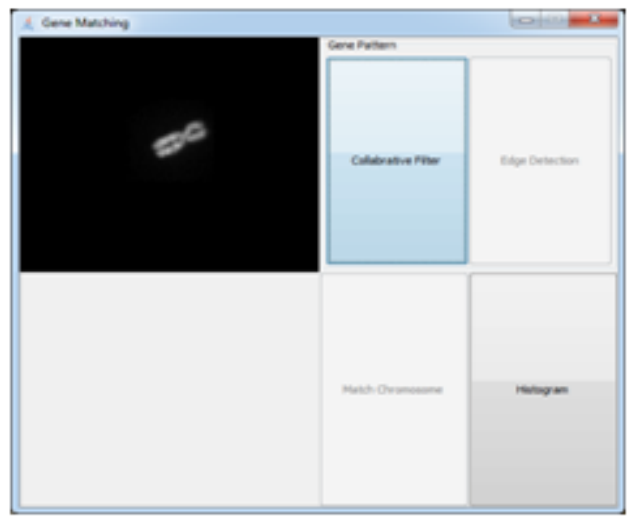

figure 5.1 Image preprocessing

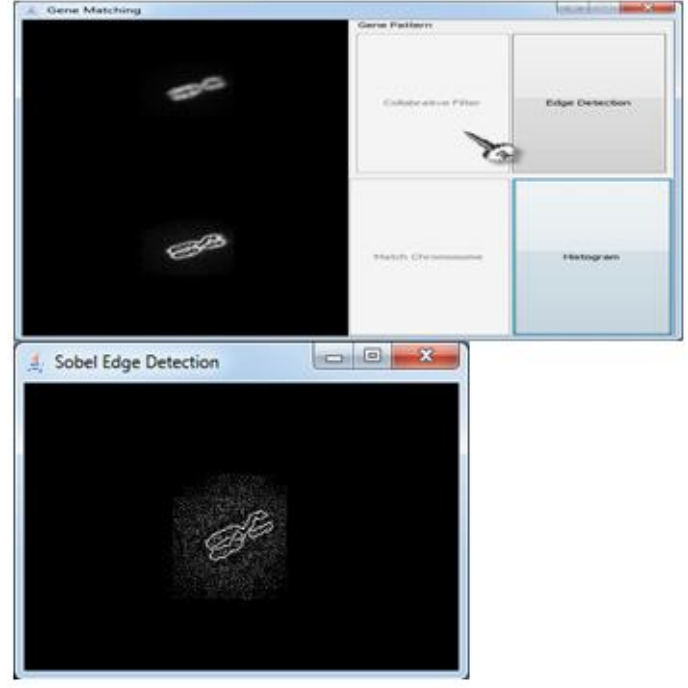

figure 5.2 Edge Detection

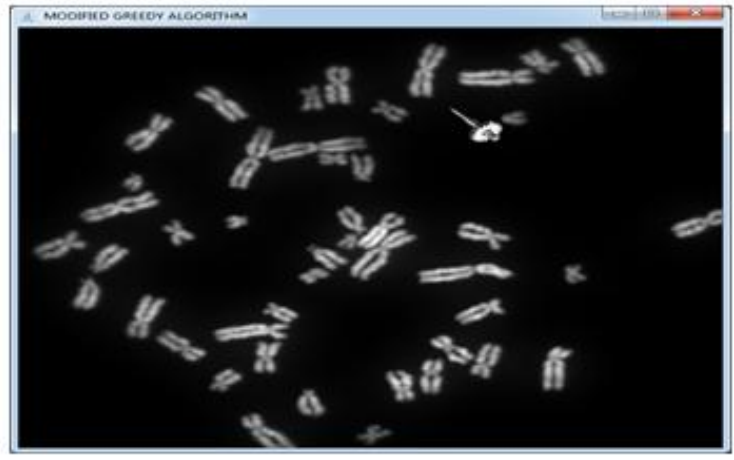

figure 5.3 Image Matching

\section{CONCLUSION}

In this system a collaborative filtering is used for preprocessing method. In the preprocessing the required information that are related to the chromosome and diseases are processed. We use edge detection method to detect the edges of the chromosome structure and so that it gets the parent chromosome. For the edge detection we use the algorithm called Sobel Edge detection. This algorithm is being used because it gives the exact outer structure when compared to that of other algorithms. Then for matching purpose the Modifies Greedy algorithm is being used to match the related chromosome. Then the bar graph is being generated. From the bar graph the possibility or the chances of the diseases for the son are displayed with the percentage of diseases. The chances are also being displayed with the color differences. Then finally the histogram can be displayed for each of the input structure that is being given. 


\section{REFERENCES}

[1] Chen H, Zhang Z. Prediction of Drug-Disease Associations for Drug Repositioning Through Drug-miRNA-Disease Heterogeneous Network. IEEE Access. 2018;6:45281-7.

[2] Sun Z, Wu Z, Zhang F, Guo Q, Chen H, Zhao J, Song D, Huang Q, Li L, Xiao J. PRAME is critical for breast cancer growth and metastasis. Gene. $2016 \mathrm{Dec}$ 5;594(1):160-4.

[3] Ritchie ME, Phipson B, Wu D, Hu Y, Law CW, Shi W, Smyth GK. limma powers differential expression analyses for RNA-sequencing and microarray studies. Nucleic acids research. 2015 Apr 20;43(7):e47-.

[4] Zhang S, Liu CC, Li W, Shen H, Laird PW, Zhou XJ. Discovery of multi-dimensional modules by integrative analysis of cancer genomic data. Nucleic acids research. 2012 Aug 8;40(19):9379-91.

[5] Lu Y, Zhou Y, Qu W, Deng M, Zhang C. A Lasso regression model for the construction of microRNA-target regulatory networks. Bioinformatics. 2011 Jul 8;27(17):2406-13.

[6] Tran DH, Satou K, Ho TB, Pham TH. Computational discovery of miR-TF regulatory modules in human genome. Bioinformation. 2010;4(8):371.

[7] Song R, Liu Q, Liu T, Li J. Connecting rules from paired miRNA and mRNA expression data sets of $\mathrm{HCV}$ patients to detect both inverse and positive regulatory relationships. InBMC genomics $2015 \mathrm{Dec}$ (Vol. 16, No. 2, p. S11). Biomed Central.

[8] Gupta S, Mazumdar SG. Sobel edge detection algorithm. International journal of computer science and management Research. 2013 Feb 2;2(2):1578-83.

[9] Carmona-Saez Chagoyen M, Rodriguez A, Trelles O, Carazo JM, Pascual-Montano A. Integrated analysis of gene expression by association rules discovery. BMC bioinformatics. 2006 Dec;7(1):54.

[10] Chen CY, Chen ST, Fuh CS, Juan HF, Huang HC. Coregulation of transcription factors and microRNAs in human transcriptional regulatory network. BMC bioinformatics. 2011 Dec;12(1):S41.

[11] Zhang S, Li Q, Liu J, Zhou XJ. A novel computational framework for simultaneous integration of multiple types of genomic data to identify microRNA-gene regulatory modules. Bioinformatics. 2011 Jun 14;27(13):i401-9.

[12] Brunet JP, Tamayo P, Golub TR, Mesirov JP. Metagenes and molecular pattern discovery using matrix factorization. Proceedings of the national academy of sciences. 2004 Mar 23;101(12):4164-9.

[13] Kim PM, Tidor B. Subsystem identification through dimensionality reduction of large-scale gene expression data. Genome research. 2003 Jul 1;13(7):1706-18.

\section{AUTHORS PROFILE}

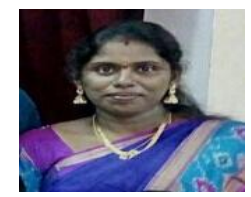

R. Kavitha, Associate Professor, Department of Computer Science and Engineering, Bharath Institute of Higher Education and Research, Chennai, India.

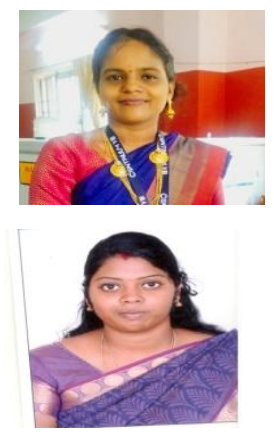

G. Kavitha, Assistant Professor, Department of Computer Science and Engineering, Bharath Institute of Higher Education and Research, Chennai, India

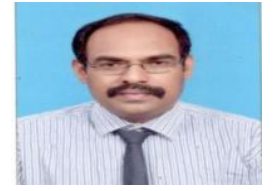

S. Sangeetha, Assistant Professor, Department of Computer Science and Engineering, Bharath Institute of Higher Education and Research, Chennai, India.

Dr. G.Michael Department of computer science and engineering ,Bharath Institute of Higher Education and Research,Chennai,Thamilnadu ,India 